\title{
CMS recent results in Heavy Flavour production and properties
}

\author{
Alexis Pompili* $\dagger$ \\ Universitá degli Studi di Bari Aldo Moro and I.N.F.N.-Sezione di Bari \\ E-mail: alexis.pompilieba.infn.it
}

Recent CMS results in the field of Heavy Flavour production and properties are reviewed.

The measurement of differential prompt production cross sections of S-wave quarkonia in pp collisions at the center-of-mass energy $\sqrt{s}=13 \mathrm{TeV}$ is reported since these are important for the study of the heavy-quark production mechanisms in quantum chromodynamics (QCD). The dependencies on transverse momentum and rapidity are investigated; comparisons with theory expectations and between different collision energies are provided as well.

Using large data samples of dimuon events, CMS has performed detailed measurements in the area of double quarkonium production since it also provides insight into the underlying mechanism of particle production. The first observation of $\mathrm{Y}(1 \mathrm{~S})$ pair production has been recently performed by exploiting proton-proton collisions at $\sqrt{s}=8 \mathrm{TeV}$. A fiducial cross section of the $\mathrm{Y}(1 \mathrm{~S})$ pair production is provided and, on its basis and the fraction of DPS contribution, an effective cross-section is also estimated.

Finally the lifetime is one of the important observables that allows to test the theoretical tools describing the physics of heavy hadrons and plays an important role in the study of non-perturbative aspects of QCD. Precise measurements of the lifetimes of the $B^{0}, B_{s}^{0}, \Lambda_{b}^{0}$ and $B_{c}^{+}$hadrons have been recently released by CMS based on the $\sqrt{s}=8 \mathrm{TeV}$ data sample. Some of the reported measurements are at the precision level of the world average for these properties.

XVII International Conference on Hadron Spectroscopy and Structure - Hadron2017

25-29 September, 2017

University of Salamanca, Salamanca, Spain

\footnotetext{
* Speaker.

${ }^{\dagger}$ On behalf of the CMS Collaboration
} 


\section{Recent results in Heavy Flavour production}

\subsection{S-wave quarkonium prompt production cross sections at $\sqrt{s}=13 \mathrm{TeV}$}

The study of quarkonium prompt production is suited to understand how quarks combine into a bound state (hadron). The well established theoretical framework of non-relativistic quantum chromo-dynamics (NRQCD) describes the quarkonium production in hadronic collisions. It is an effective theory that assumes that the production mechanism can be factorized in two steps. In the first, a heavy quark-antiquark pair is produced in a given spin and orbital angular momentum state, either in a color-singlet or color-octet configuration. The corresponding parton-level cross sections, called short-distance coefficients (SDCs), are functions of the kinematics of the state and can be calculated perturbatively, presently up to next-to-leading order (NLO). In the second step, the quark-antiquark pairs bind into the final quarkonium states through a non-perturbative hadronization process, with transition probabilities determined by process-independent long-distance matrix elements (LDMEs). The LDMEs are presently not calculable and must be obtained through fits to experimental data.

Given the several measurements of production cross-sections and polarizations performed by the LHC experiments with Run-I data[1], the theoretical developments to explain both crosssections and polarizations results get reasonable agreement with data[2] once excluding low- $p_{T}$ data $\left(p_{T} / M_{\text {hadron }}>3\right)$ and assert that the unpolarized color-octet $\left({ }^{1} S_{0}\right)$ contribution dominates the production[3]. Instead, from previous expectations, the color-octet ${ }^{3} S_{1}$ was thought to dominate, thus implying a strong transverse polarization of the mesons relative to their direction of motion at large $p_{T}$ values. The crucial questions about the validity domain of NRQCD at NLO, about what happens at higher $p_{T}$ values and about the relative weights of the LDMEs require further, both theoretical and experimental, investigation that can be addressed with new data.

LHC Run-II data (at increased center-of-mass energy and production cross-sections, and with higher integrated luminosity) will play a crucial role in measuring the production cross sections and polarizations of S- and P-wave quarkonium states while extending the $p_{T}$-reach and thus testing the validity domain of NRQCD. Moreover the measurements performed at $13 \mathrm{TeV}$ also provide the opportunity to test the $\sqrt{s}$-dependence of the cross sections and to check the validity of the factorization hypothesis and LDME universality implied in NRQCD (while using perturbative calculations appropriate to the two different center-of-mass energies).

CMS[4] has recently measured differential prompt production cross sections of $J / \psi$ and $\psi(2 S)$ charmonium and $\Upsilon(n S)(n=1,2,3)$ bottomonium states in proton-proton collisions at $\sqrt{s}=$ $13 \mathrm{TeV}$, with data corresponding to an integrated luminosity of $2.3 \mathrm{fb}^{-1}$ for the $J / \psi$ and $2.7 \mathrm{fb}^{-1}$ for the other mesons. The five quarkonium states are reconstructed in the dimuon decay channel, for dimuon rapidity $|y|<1.2$. The new analysis used the same technique previously employed studying Run-I data[5][6]. The double differential prompt production cross-section, times the branching fraction of the meson in two muons, are calculated, for each state, in several dimuon $p_{T}$ and $|y|$ bins according to the following formula:

$$
\mathscr{B}\left(q \bar{q} \rightarrow \mu^{+} \mu^{-}\right) \times \frac{d^{2} \sigma^{q \bar{q}}}{d p_{T} d y}=\frac{\mathscr{N}^{q \bar{q}}\left(p_{T}, y\right)}{\mathscr{L} \Delta p_{T} \Delta y} \cdot\left\langle\frac{1}{\varepsilon\left(p_{T}, y\right) A\left(p_{T}, y\right)}\right\rangle
$$


namely obtained by dividing, by the integrated luminosity $\mathscr{L}$, the number of prompt candidates $\mathscr{N}^{q \bar{q}}\left(p_{T}, y\right)$ extracted in a bin of widths $\left(\Delta p_{T}, \Delta y\right)$ and weighted by the average of the product of the inverse efficiency and acceptance for all the candidates in the bin. Efficiencies are derived by means of a data-driven approach while acceptances from simulations. Non-prompt components for S-wave charmonia (due to decays of beauty hadrons) are separated by using two-dimensional simultaneous fits to the pseudo-proper decay length and to the invariant mass of the dimuon, whereas directly produced quarkonia are not distinguished from feed-down decays of heavier quarkonium states (note that $\psi(2 S)$ is feed-down free). The results at $\sqrt{s}=13 \mathrm{TeV}$ are presented in Fig.1 together with a comparison to theoretical expectations and to the $\sqrt{s}=7 \mathrm{TeV}$ measurements in order to check the scaling in cross-section due to the center-of-mass energy increase. Cross sections at $13 \mathrm{TeV}$ are a factor $2-3$ larger than the corresponding values at $7 \mathrm{TeV}$ and slowly increasing as a function of the $p_{T}$. An increase of this order is expected from the evolution of parton distribution functions (verified using PYTHIA8). These results will contribute to consolidate the underlying hypothesis of NRQCD and provide further input to constrain the theory parameters.
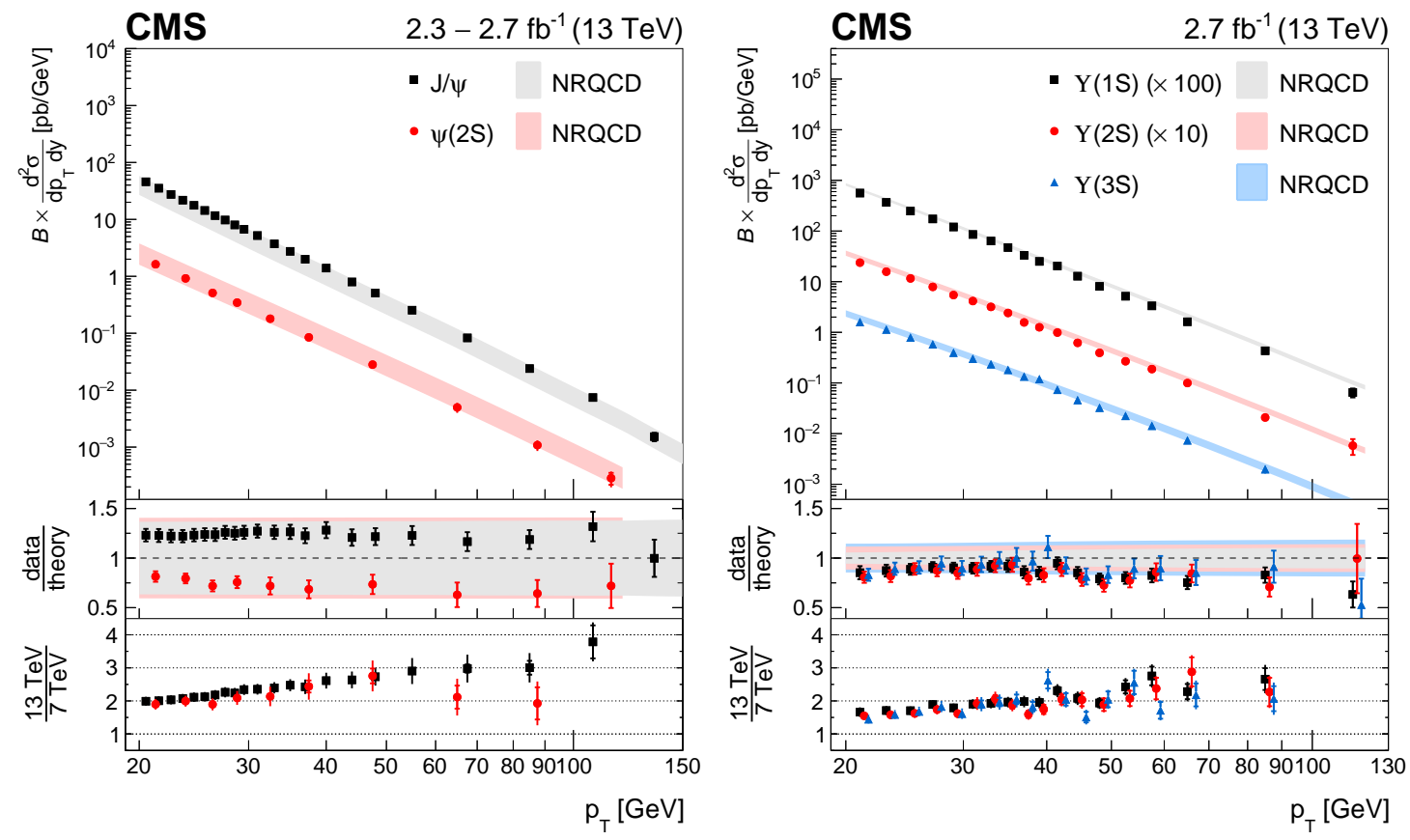

Figure 1: From Ref.[4]. The measured double-differential cross sections times branching fractions of the prompt $J / \psi$ and $\psi(2 S)$ (left) and $\Upsilon(n S)$ (right) mesons, assuming their dimuon decays to be isotropic, are given as a function of $p_{T}$ for $|y|<1.2$ and compared to NLO NRQCD predictions (shaded bands). The inner vertical bars on the data points represent the statistical uncertainty, while the outer bars show the statistical and systematic uncertainties, including the integrated luminosity uncertainty of $2.3 \%$, added in quadrature. The middle panels show the ratios of measurement to theory, where the vertical bars depict the total uncertainties in the measurement. The widths of the bands represent the theoretical uncertainty, added in quadrature with the uncertainties in the dimuon branching fractions. The lower panels show the ratios of cross sections measured at $13 \mathrm{TeV}$ and $7 \mathrm{TeV}$, with their uncertainties being treated as uncorrelated. 


\subsection{First prompt double $\Upsilon(1 S)$ observation at $\sqrt{s}=8 \mathrm{TeV}$}

The measurement of heavy quarkonium pair production in pp collisions provides further insight into the underlying mechanism of particle production. It probes specific mechanism of $c \bar{c} c \bar{c}$ and $b \bar{b} b \bar{b}$ systems production and subsequent transformation to two mesons, namely it probes the distribution of gluons in a proton since their production should be dominated by gluon-gluon interactions. According to the description by parton models of production in QCD, in a single hadronhadron collision two partons often undergo a single interaction (Single Parton Scattering: SPS). The SPS mechanism can be described by NRQCD. It is also possible that multiple distinct parton interactions occur, the simplest case being the Double Parton Scattering (DPS), namely 2 distinct parton-parton collisions in the same pp interaction. Because of the high parton flux and the high center-of-mass energy at LHC, the pair production can be potentially sensitive to DPS. These nontrivial contributions expected from DPS are difficult to be addressed within the perturbative QCD framework and thus cannot be modelled by current NRQCD predictions.

Theoretical predictions for DPS require a factorization theorem for the cross section, in order to separate the two short-distance collisions from the long-range physics of the incoming protons. The partonic cross section of the hard scatterings is perturbatively calculable whereas the momenta of the quarks and gluons inside the proton are described by non-perturbative parton distribution functions (PDFs), which must be modeled or extracted from data. If the two hard scatterings are independent and the two incoming partons in each proton are completely independent, the DPS cross section simplifies[7], in the case of double quarkonia in the final state, to

$$
\sigma_{D P S}=\frac{1}{2} \frac{\sigma_{\text {single-quarkonium }}^{2}}{\sigma_{e f f}}
$$

This approximation leaves a single non-perturbative parameter, the effective cross-section $\sigma_{e f f}$, which only affects the total DPS rate. It can be thought as characterizing the transverse area of the hard partonic interactions and it is expected to be independent of the final state and the center-ofmass energy (assuming PDFs not correlated). From a fit to the differential cross section measurements of $J / \psi$ pair production by CMS[8] with $\sqrt{s}=7 \mathrm{TeV}$ data, Lansberg and Shao[9] estimate an effective cross section of $8.2 \mathrm{mb}$.

CMS has recently reported[10] the first observation of prompt $\Upsilon(1 S)$ pair production from a data sample at $\sqrt{s}=8 \mathrm{TeV}$ corresponding to an integrated luminosity of $20.7 \mathrm{fb}^{-1}$, and also the estimation of $\sigma_{e f f}$ using this result, along with theoretical predictions.

Candidate $\Upsilon$ events are required to have four or more muons that pass the first-level muon trigger, of which at least three must be identified as muons by the High Level Trigger. Details of the offline selection can be found in[10]. To suppress contributions from pileup events, all four muons are required to be associated with a common vertex having a $\chi^{2}$ probability larger than $5 \%$. To ensure a nearly uniform single-muon acceptance and a well-defined kinematic region, the muon candidates must have $p_{T}^{\mu}>3.5 \mathrm{GeV}$ and $\left|\eta^{\mu}\right|<2.4$. In order to measure the cross section in a well-defined kinematic region, both $\Upsilon$ candidates must have $\left|y^{\Upsilon}\right|<2.0$ thus defining the fiducial region in terms of rapidity. At the end of the offline selection the $4 \%$ of the events that contain more than one $\Upsilon$ candidate are discarded. After all selection criteria are applied, a total of $313 \Upsilon \Upsilon$ candidates are found. 
To extract the signal yield of $\Upsilon(1 S) \Upsilon(1 S)$ candidates, a two-dimensional extended unbinned maximum-likelihood (UML) fit to the invariant masses of the two reconstructed dimuon combinations is used. Between them an higher $\left(M_{\mu \mu}^{1}\right)$ and a lower $\left(M_{\mu \mu}^{2}\right)$ invariant masses are specified in order to resolve the ambiguity of having a dimuon pair in which an $\Upsilon(2 S)$ candidate can appear. The 2D fit considers five categories of events: 1) $(1 S) \Upsilon(1 S)$ signal candidates, 2) $\Upsilon(2 S) \Upsilon(1 S)$ candidates, 3) $\Upsilon(1 S)$ plus 2 unassociated muons, 4) $(2 S)$ plus 2 unassociated muons, 5) fully combinatorial background (4 unassociated muons). Other categories are marginal; for instance there is no indication of $\Upsilon(2 S) \Upsilon(2 S)$ candidates. The modelling of these components are described in detail in[10]. The projections of the 2D ML fit onto the higher- and lower-mass are presented in Fig.2. The extracted yields obtained by this procedure are $\mathscr{N}_{\text {signal }}=38 \pm 7$ and $N_{\Upsilon(2 S) \Upsilon(1 S)}=13_{-5}^{+6}$. The corresponding statistical significance of the former signal is found to be well above 5 standard deviations whereas, for the latter, it is estimated to be 2.6 standard deviations.

Assuming that both mesons decay isotropically, the $\Upsilon(1 S) \Upsilon(1 S)$ production cross section in the fiducial region is obtained by the expression:

$$
\sigma_{\text {fid }}=\frac{\mathscr{N}_{\text {signal }}}{\mathscr{L}\left[\mathscr{B}\left(\Upsilon(1 S) \rightarrow \mu^{+} \mu^{-}\right)\right]^{2}} \cdot\left\langle\frac{1}{\varepsilon_{i} \cdot A_{i}}\right\rangle
$$

where $\mathscr{L}$ is the integrated luminosity, $\mathscr{B}$ the world-average branching fraction, and the correction factor is the inverse product of per-event efficiencies and acceptances averaged over the events that pass the selection criteria; this factor accounts for the inefficiencies stemming from muon reconstruction and identification, and trigger requirements, as well as for the fraction of events lost because of the muon acceptance criteria. Calculation of corrections on an event-by-event basis using measured momenta of $\Upsilon(1 S)$ and muons (with a data-embedding method into simulations) minimizes the model dependency of corrections. Finite $\mu^{+} \mu^{-}$resolution can distort measured momenta (mostly close to the borders of the kinematic acceptance of the detector), thus scaling factors are determined for both SPS and DPS simulated samples (98\% and $96 \%$ respectively). The resulting estimate of the fiducial cross section is $\sigma_{\text {fid }}=[68.8 \pm 12.7($ stat $) \pm 7.4($ syst $) \pm 2.8(\mathscr{B})] \mathrm{pb}$. Assum-
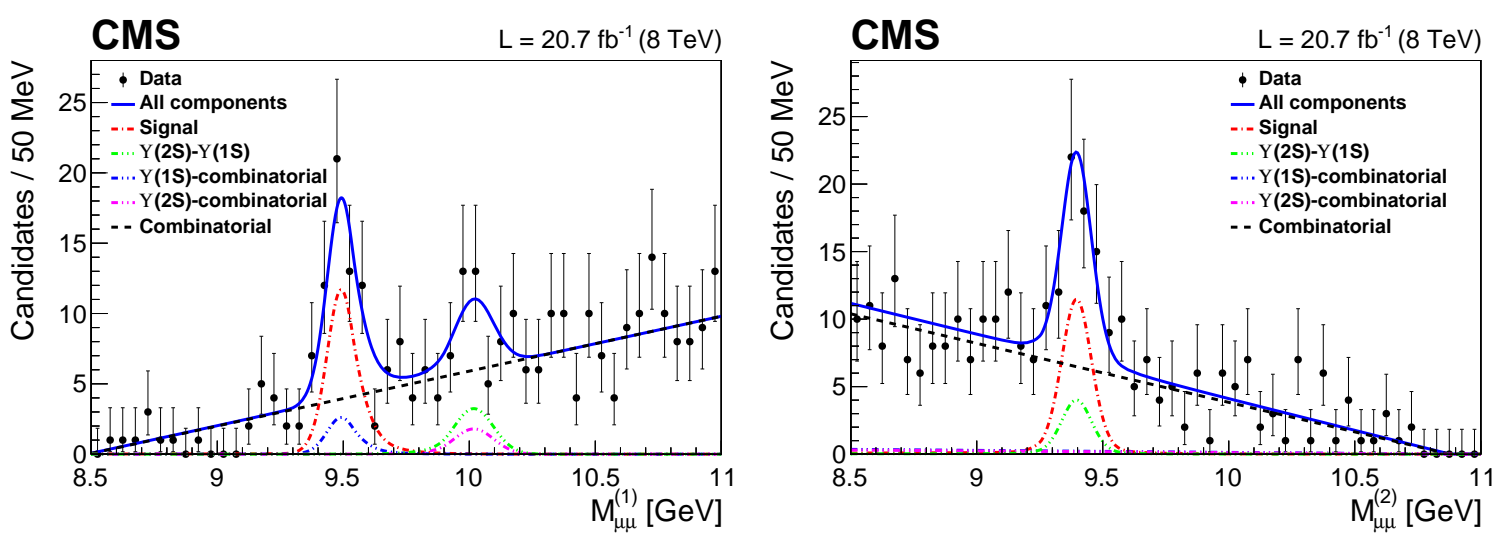

Figure 2: From Ref.[10]. Invariant mass distributions of the higher-mass muon pair (left) and the lowermass muon pair (right). The data are shown by the points. The different curves show the contributions of the various event categories from the fit, as defined in the text. 
ing the $\Upsilon(1 S)$ mesons are produced with different (extreme) polarizations, the cross section varies in the range $-38 \%$ to $+36 \%$ with respect to the measured value. This, as well as the other systematic uncertainties are discussed in detail in Ref.[10].

The effective cross section can be estimated exploiting the previous $\sigma_{\text {fid }}$ result:

$$
\sigma_{e f f}=\frac{1}{2 f_{D P S} \cdot \sigma_{f i d}} \cdot \frac{[\sigma(\Upsilon)]^{2}}{\left[\mathscr{B}\left(\Upsilon(1 S) \rightarrow \mu^{+} \mu^{-}\right)\right]^{2}}
$$

where $f_{D P S}$ is the fraction of DPS contribution and $\sigma(\Upsilon)$ is the cross section for single- $\Upsilon(1 S)$ production that was measured by CMS[11] and has been extrapolated to the fiducial region $\left(\left|y^{\mathrm{Y}}\right|<\right.$ 2.0) used to estimate $\sigma_{\text {fid }}$. In this analysis the statistics is not enough to extract the DPS fraction which can be typically estimated either as a residual of the SPS prediction or as a result of a fit to the absolute rapidity difference between the two quarkonia (large $|\Delta y|$ values are possible for DPS production). By assuming a fraction between $10 \%$ and $30 \%$ one gets $2.2 \mathrm{mb}<\sigma_{\text {eff }}<6.6 \mathrm{mb}$. This estimate is a) in agreement with the values from heavy quarkonium measurements ( 2 to $8 \mathrm{mb}$ ), and b) smaller than that from multijet final states (12 to 20mb)[12].

Since quarkonium final states are dominantly produced by gluon-gluon interactions while jet related channels are produced by quark-antiquark or quark-gluon interactions, this trend might indicate that the transverse distance among gluons in the proton is smaller than among quarks or quarks and gluons.

\section{Recent results in Heavy Flavour properties}

CMS[13] has recently provided, in proton-proton collisions at $\sqrt{s}=8 \mathrm{TeV}$, with data corresponding to an integrated luminosity of $19.7 \mathrm{fb}^{-1}$, precise lifetime measurements of b-hadrons reconstructed in final states with a $J / \psi$ decaying into two muons. The following decay channels have been used: $B^{0} \rightarrow J / \psi K^{*}(892)^{0}, B^{0} \rightarrow J / \psi K^{0} s, B_{s}^{0} \rightarrow J / \psi \pi^{+} \pi^{-}, B_{s}^{0} \rightarrow J / \psi \phi(1020)$, $\Lambda_{b}^{0} \rightarrow J / \psi \Lambda^{0}$ and $B_{c}^{+} \rightarrow J / \psi \pi^{+}$.

$B^{+} \rightarrow J / \psi K^{+}$is the simplest decay mode with the highest yield and is used as reference channel; specifically it is used for overall calibration and specific systematic studies. It is also used for the measurement of the $B_{c}^{+}$lifetime as discussed later.

\subsection{Reconstruction of $b$-hadrons and lifetime measurement method}

Details of the reconstruction of b-hadrons and their signal extraction can be found at Ref.[13]. As an example the signal of $B_{s}^{0}$ and $B_{c}^{+}$reconstructed candidates are reported in Fig.3.

The proper decay time of the $b$-hadron candidate times the speed of light is

$$
c t=c \frac{L}{\beta \gamma c}=c M_{P D G} \cdot \frac{L}{|\vec{p}|}=c M_{P D G} \cdot \frac{L_{x y}}{\left|\overrightarrow{p_{T}}\right|}
$$

where $L_{x y}$ is the transverse flight distance from the primary vertex (production vertex for the $b$ hadron) to the decay vertex and $M_{P D G}$ is the world average mass value of a $b$-hadron. 

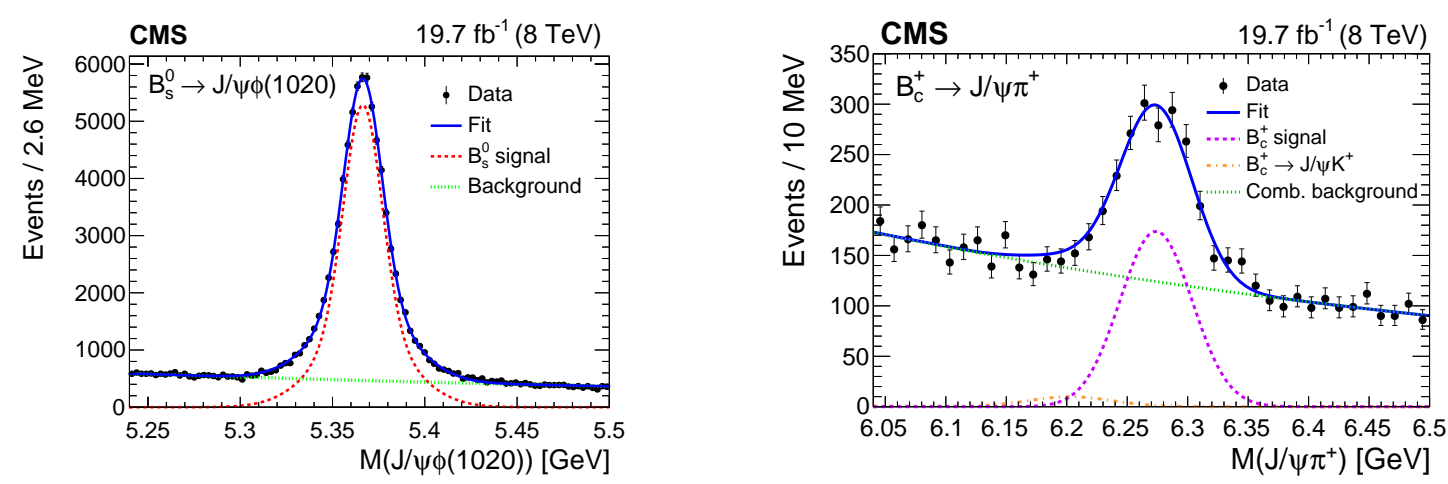

Figure 3: From Ref.[13]. Invariant mass distribution for $B_{s}^{0}$ candidates reconstructed from $J / \psi \phi$ decays (left plot) and for $B_{c}^{+}$candidates reconstructed from $J / \psi \pi^{+}$decays (right plot). The curves are fit projections to the data, with the solid (blue) lines representing the total fit, the dashed (red) lines the signal component, the dotted (green) line the combinatorial background. Moreover, in the right plot, the dashed-dotted (orange) line represents the contribution from suppressed $B_{c}^{+} \rightarrow J / \psi K^{+}$decays.

The proper time resolution $\sigma_{c t}$ is determined for each candidate by propagating the uncertainties on displacement (position of vertices) and on momentum. The distortion of the $c t$-distribution is due to a sum of effects generated by reconstruction and selection criteria; it is taken into account by the efficiency term $\varepsilon(c t)$ determined from simulations of individual decay modes. The efficiency turn-on region is discarded by selecting $c t>200 \mu \mathrm{m}\left(>100 \mu \mathrm{m}\right.$ for $\left.B_{c}^{+}\right)$; the $\varepsilon(c t)$ is parametrized by an inverse power function for $c t>200 \mu \mathrm{m}\left(>100 \mu \mathrm{m}\right.$ for $\left.B_{c}^{+}\right)$. The efficiency for the $B_{s}^{0} \rightarrow J / \psi \phi$ channel (in Fig.4, left plot) is defined as the $c t$ distribution of the selected candidates after reconstruction divided by the sum of the two exponentials generated with the theoretical decay model used in the simulation (the two involved decay times are those associated to the light and heavy mass eigenstates).
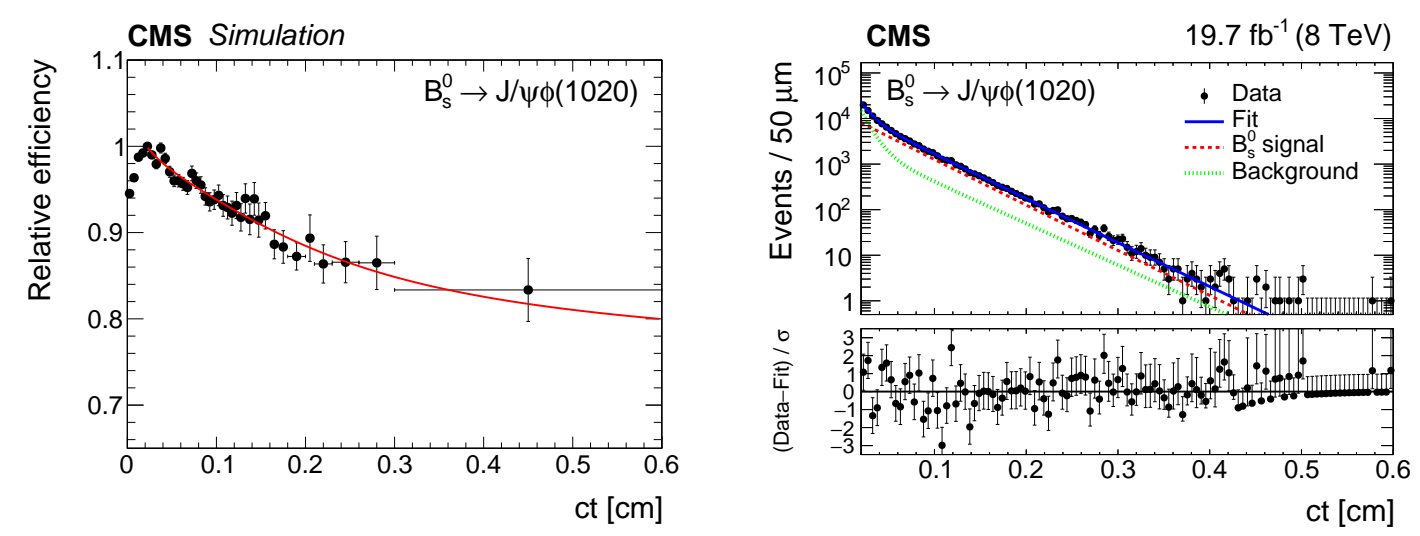

Figure 4: From Ref.[13]. Left plot: the combined reconstruction and selection efficiency from simulation versus $c t$ with a superimposed fit to an inverse power function for $B_{s}^{0} \rightarrow J / \psi \phi$; the efficiency scale is arbitrary (only the shape is relevant). Right plot: $c t$ distribution for $B_{s}^{0}$ candidates reconstructed from $J / \psi \phi$ decays. The curves are projections of the fit to the data; the representation scheme is the same used in Fig.3. The bottom panel shows the fit pulls bin-by-bin. 
For the $B^{+}, B^{0}$ and $\Lambda_{b}^{0}$ hadrons, a three-dimensional Unbinned Maximum Likelihood fit to the data is performed. An extended UML fit is used for $B_{s}^{0}$ modes. The three input variables are the $b$-hadron mass, $c t$ and $c t$ uncertainty $\left(\sigma_{c t}\right)$. The signal $c t$ distribution is modelled by an exponential function, convoluted with a gaussian resolution function with the per-candidate width taken from the $\sigma_{c t}$ distribution, and then multiplied by a function describing the reconstruction and selection efficiency $\varepsilon(c t)$. The backgrounds are described by an heuristic sum of exponential functions convoluted with the resolution. Further details about the lifetime estimation by the fit can be found in Ref.[13]. The lifetime measurement in the reference $B^{+}$channel is $c \tau=[490.9 \pm 0.8$ (stat) $] \mu \mathrm{m}$ (to be compared with the average PDG-2016 value [491.4 \pm 1.2$] \mu \mathrm{m}$ ). The $\Lambda_{b}^{0}$ lifetime is also in good agreement with the current world average value. The same holds for the two independent measurements of $B^{0}$ lifetime (from the two used decay channels) that are fully consistent between each other. The lifetime results of $B^{0}$ and $\Lambda_{b}^{0}$ are reported in Tab.1, in the last subsection.

\section{$2.2 B_{s}^{0}$ lifetime}

$B_{s}^{0}$ mesons undergo rapid spontaneous transitions between particle and antiparticle states; the system is described by heavy/light, $B_{H} / B_{L}$, mass eigenstates characterized by sizable differences: $\Delta m_{s}=m_{H}-m_{L} \simeq 17.76 \pm 0.02 \mathrm{ps}^{-1}$ and $\Delta \Gamma / \bar{\Gamma}=\Gamma_{H}-\Gamma_{L} / \bar{\Gamma} \simeq-0.12 \pm 0.01$ (where $\bar{\Gamma}=\Gamma_{H}+$ $\Gamma_{L} / 2$ ). In the absence of sizable CP violation in the $B_{s}^{0}$ system, heavy/light eigenstates correspond to $\mathrm{CP}$-odd/even eigenstates respectively.

The final state in the decay $B_{s}^{0} \rightarrow J / \psi \pi^{+} \pi^{-}$is dominated by $f_{0}(980)$, making it a CP-odd state; thus the measured lifetime gives direct access to $\tau_{H}$. The related reconstructed signal has smaller yield and larger background with respect to the $B_{s}^{0} \rightarrow J / \psi \phi(1020)$ decay. The final state in the latter decay mode is an admixture of one CP-odd and two CP-even eigenstates; thus the $c t$ distribution consists in two exponentials and the measured lifetime is an effective lifetime $\left(\tau_{\text {eff }}\right)$ that is a weighted average of $\tau_{H}$ and $\tau_{L}$. Details can be found in Ref.[13]. The lifetime fit projections to the $c t$ distribution for the $B_{s}^{0} \rightarrow J / \psi \phi$ are presented in Fig.4 (right plot).

The present measurement is complementary to the weak mixing phase $\phi_{s}$ analysis[14] where the CP-odd/even components are disentangled by an angular analysis.

The bias caused by the contamination of non-resonant $B_{s}^{0} \rightarrow J / \psi K^{+} K^{-}$decays (CP-odd final state) is considered as a systematic uncertainty. The $S$-wave fraction is assigned the value measured in Ref.[14] after the application of the needed correction to account for different trigger and selection criteria in the two analyses $\left(f_{s}=1.2_{-0.7}^{+0.9} \%\right)$.

Finally it is worthy to mention that the $B_{H} / B_{L}$ relative fraction evolves in time and thus the cut $c t>200 \mu$ m modifies the relative composition; consequently the $c \tau_{\text {eff }}$ must be properly corrected.

The results for $c \tau_{H}$ and $c \tau_{e f f}$ is reported in Tab.1; by combining these two measured lifetimes it is possible to determine $\tau_{L}$ as well.

\section{$2.3 B_{c}^{+}$lifetime}

The weak decay of the $B_{c}^{+}$can occur through either the $\mathrm{b}$ or the c quark (this implies shorter lifetimes than other B mesons) or through an annihilation process (predicted to contribute $10 \%$ of the decay width). The $B_{c}^{+}$lifetime is measured exploiting the ratio method previously used by $\mathrm{LHCb}[15]$, in which the measured difference in total widths between the $B_{c}^{+}$and $B^{+}$mesons is used 
in combination with the precisely known $B^{+}$lifetime. The same reconstruction algorithm and selection criteria are used for both decays $B_{c}^{+} \rightarrow J / \psi \pi^{+}$and $B^{+} \rightarrow J / \psi K^{+}$. This method, in which the systematic uncertainties are reduced, was adopted for $B_{c}^{+}$in view of the larger background contamination and the smaller lifetime to be measured.

The decay time distribution for the signal, either $B_{c}^{+}$or $B_{c}^{+}$, can be expressed as the product

$$
N_{B}(c t)=\left[E\left(c t ; c \tau_{B}\right) \otimes G(c t)\right] \cdot \varepsilon_{B}(c t)
$$

where $E\left(c t ; c \tau_{B}\right) \equiv e^{-c t / c \tau_{B}}$ is an exponential decay function and $\varepsilon_{B}(c t)$ is the $c t$-efficiency function. Thus the ratio of the yields of $B_{c}^{+}$and $B^{+}$candidates, at a given proper time, can be expressed as

$$
\frac{N_{B_{c}^{+}}(c t)}{N_{B^{+}}(c t)} \equiv \mathscr{R}(c t)=\frac{\left[E\left(c t ; \tau_{B_{c}^{+}}\right) \otimes G(c t)\right] \cdot \varepsilon_{B_{c}^{+}}(c t)}{\left[E\left(c t ; \tau_{B^{+}}\right) \otimes G(c t)\right] \cdot \varepsilon_{B^{+}}(c t)} \simeq R_{\varepsilon}(c t) \cdot e^{-\Delta \Gamma t}
$$

where the approximation holds after having verified that the ratio is negligibly affected by the time resolution, $R_{\varepsilon}(t)$ just denotes the ratio of $c t$-efficiencies and

$$
\Delta \Gamma=\Gamma_{B_{c}^{+}}-\Gamma_{B^{+}}=\frac{1}{\tau_{B_{c}^{+}}}-\frac{1}{\tau_{B^{+}}}
$$

The $\Delta \Gamma$ estimation is obtained by fitting with an exponential function the ratio $\mathscr{R}(c t)$. Subsequently the $\Delta \Gamma$ result is converted to $B_{c}^{+}$lifetime by using the world average $B^{+}$lifetime. The result is reported in Tab.1. It is in agreement with recent LHCb measurements whereas is larger than those at Tevatron. Precise measurements of the $B_{c}^{+}$lifetime allows to test various theoretical models[16].

\subsection{Lifetime results}

All the precise lifetime measurements are summarized in Tab.1. A full discussion about the systematic uncertainties, either common or channel-specific, can be found in Ref.[13].

\begin{tabular}{ll}
\hline Decay mode & $c \tau[\mu \mathrm{m}]$ \\
\hline \hline$B^{0} \rightarrow J / \psi K^{*}(892)^{0}$ & $453.0 \pm 1.6($ stat $) \pm 1.5$ (syst) \\
$B^{0} \rightarrow J / \psi K^{0} s$ & $457.8 \pm 2.7($ stat $) \pm 2.7$ (syst) \\
$\Lambda_{b}^{0} \rightarrow J / \psi \Lambda^{0}$ & $442.9 \pm 8.2$ (stat) \pm 2.7 (syst) \\
$B_{s}^{0} \rightarrow J / \psi \pi^{+} \pi^{-}$ & $502.7 \pm 10.2($ stat $) \pm 3.2\left(\right.$ syst $\left[c \tau_{H}\right]$ \\
$B_{s}^{0} \rightarrow J / \psi \phi(1020)$ & $443.9 \pm 2.0($ stat $) \pm 1.2$ (syst) $\left[c \tau_{e f f}\right]$ \\
$B_{c}^{+} \rightarrow J / \psi \pi^{+}$ & $162.3 \pm 8.2($ stat $) \pm 4.7$ (syst) $\pm 0.1\left(\tau_{B^{+}}\right)$ \\
\hline
\end{tabular}

Table 1: Lifetime measurements performed in Ref.[13]. For $B_{s}^{0}: c \tau_{L}=[419.7 \pm 6.3] \mu \mathrm{m}$ is also estimated.

\section{Conclusions}

Recent CMS results have been reported in the area of heavy flavour production and properties. In particular three measurements have been reviewed here.

(a) Differential prompt production cross sections of five S-wave quarkonia, reconstructed in the dimuon decay channel, have been measured, with a sub-sample of data collected at the centerof-mass energy of $13 \mathrm{TeV}$, as a function of rapidity and transverse momentum, and compared to 
theoretical expectations from NRQCD. Cross sections at $13 \mathrm{TeV}$ are found to be a factor $2-3$ larger than the corresponding values at $7 \mathrm{TeV}$ and slowly increasing with the transverse momentum.

(b) The first observation of the pair production of $\Upsilon(1 S)$, fully reconstructed via their dimuon decays, has been reported by studying the full data sample collected at the center-of-mass energy of $8 \mathrm{TeV}$. The prompt production cross section of $\Upsilon(1 S)$ pairs has been measured in a fiducial acceptance rapidity region; an effective cross section is also estimated and it is found in agreement with the values from heavy-quarkonium measurements but smaller than that from multijet studies.

(c) The $\sqrt{s}=8 \mathrm{TeV}$ data sample has been also exploited to measure the lifetimes of the $B^{0}, B_{s}^{0}$, $\Lambda_{b}^{0}$ and $B_{c}^{+}$hadrons by using their fully reconstructed decays with a $J / \psi$ meson in the final state. The precision from each of the used decay channels is as good as or better than previous measurements. All results are in agreement with current world-average values. The measurement of $B_{c}^{+}$ lifetime, performed with respect to the $B^{+}$to reduce the systematic uncertainty, is in agreement with results from $\mathrm{LHCb}$, thus confirming a longer lifetime than found by Tevatron experiments.

\section{References}

[1] For a full and updated bibliography see Ref.[4]. For S-wave quarkonia: the CMS measurements of differential production cross-sections at 7TeV are provided in Ref.[5][6]; the CMS measurements of polarizations are presented in Phys. Lett. B 727 (2013) 382 and Phys. Rev. Lett. 110 (2013) 081802.

[2] See, among others, P. Faccioli et al., Phys. Lett. B 736 (2014) 98 and G.T. Bodwin et al., Phys. Rev. Lett. 113 (2014) 022001.

[3] Recently CMS Collaboration (Phys. Lett. B 761 (2016) 31) has measured $\Upsilon(n S)$ polarizations as a function of charged particle multiplicity into $p_{T}$ bins. No influence of the surrounding medium was detected since no significant changes from low- to high-multiplicity pp collisions were observed (but large uncertainties preclude definite statements for $n=2,3$ ).

[4] CMS Collaboration, e-print arXiv:1710.11002, submitted to Phys. Lett. B; preliminary results shown at the conference are contained in the Physics Analysis Summary CMS-PAS-BPH-15-005.

[5] CMS Collaboration, Phys. Rev. Lett. 114 (2015) 191802.

[6] CMS Collaboration, Phys. Lett. B 749 (2015) 14.

[7] See S.P. Baranov et al., Phys. Lett. B 746, (2015) 100, and references therein.

[8] CMS Collaboration, JHEP 09 (2014) 094.

[9] J.-P. Lansberg and H.-S. Shao, Phys. Lett. B 751 (2015) 479.

[10] CMS Collaboration, JHEP 05 (2017) 013.

[11] CMS Collaboration, Phys. Lett. B 727 (2013) 101.

[12] See also the review from S. Palestini in these proceedings.

[13] CMS Collaboration, e-print arXiv:1710.08949, submitted to Eur. Phys. J. C; preliminary results shown at the conference are in the Physics Analysis Summary CMS-PAS-BPH-13-008.

[14] CMS Collaboration, Phys. Lett. B 757 (2016) 97.

[15] LHCb Collaboration, Phys. Lett. B 742 (2015) 29.

[16] For details see Ref.[13] and references therein. 